\title{
The Relationship Between Cross Cultural Adaptation and Turnover Intention: A Study of Lao Employees in Foreign Enterprises
}

\author{
Somchit Hongvichit ${ }^{1}$ \\ ${ }^{1}$ Glorious Sun School of Business and Management, Dong Hua University, Shanghai, China \\ Correspondence: Somchit Hongvichit, Glorious Sun School of Business and Management, Dong Hua University, \\ Shanghai, China. Tel: 86-137-6132-8644. E-mail: somchit@163.com
}

Received: June 9, 2015

Accepted: June 30, 2015

Online Published: July 25, 2015

doi:10.5539/ibr.v8n8p164

URL: http://dx.doi.org/10.5539/ibr.v8n8p164

\begin{abstract}
With a variety of local advantages, a large number of foreign investments into Laos, Lao's employees in foreign enterprise began to be concerned about their turnover. However, research in this part is still blank. Since most of the researches based on the employees and the organization are belong to the same cultural background, we try to find out if the cultural factor also is one of important factor which impact to local employee's turnover intention. The relationship between cross cultural adaptation and turnover intention is main discussing topic on this study, local employee in foreign enterprise as the research subjects for this study. Based on survey data to calculate the cross-cultural adaptation index and turnover intention index, then regression analysis to find out the relationship of both index. The results proved that cross cultural adaptation and turnover intention was negatively correlated. This means cross-cultural adaptation is not a small obstacle but it's one of the most important factors to determine Laos's employees who work in foreign enterprises to leave or stay.
\end{abstract}

Keywords: turnover intention, cross cultural adaptation, turnover intention index, cross cultural adaptation index

\section{Introduction}

Lao People's Democratic Republic was established in 1975, economic reform and opening up in 1983 and overall economic development in recent years. With a variety of local advantages (infrastructure conditions, low labor costs, Policy advantage ... etc.), a large number of foreign investment into Laos, Lao employee in foreign enterprise turnover issue began to be concerned about. However, research in this part is still blank.

Previous studies of employee turnover issue focused on job satisfaction, organizational commitment, organizational support...etc. as main measure. Practice a lot of achievements in this field have also been applied in. But most of the researches based on the employees and the organization it belongs to the same cultural background. Therefore, what would happen if employee and the organization come from different cultural backgrounds? Are the traditional theory and research methods are sufficient to explain all the phenomena? Or some factors more prominent?

Based on the above ideas, local employee in foreign enterprise as the research subjects for this study. To investigate if the employee and the organization were from different cultural backgrounds, the cultural factor will have an impact on employee turnover intention or not? Since previous studies have not been studied turnover intention of Lao employee, especially Lao employee in foreign enterprise. Therefore, this is a new attempt. In order to better reflect the impact of cultural factor to turnover intention, these papers introduce the concept of cross-cultural adaption and analyze the relationship with turnover intention.

\section{Literature Review}

\subsection{The Concept of Cross-Cultural Adaption Ability}

Redfield el al. (1936) defined cross cultural adaptation as "Composed by individual and between two group which from different cultures. It had persistent and direct cultural contacts, resulting in one or both of the original cultural patterns change phenomenon." Zhao Qing (2010) believes that cross-cultural adaption is the progress of integration and coordination between two different cultural groups.

Guo Ying el al. (2005) believe that adaptation is the environment and people coordinate with each other to achieve a balanced state. Zhou Lin Gang el al. (2005) mentioned "When the role of individual and the 
environment changed dramatically will break the original balance". The further explanation by Yu Wei and Zheng Gang (2005) mentioned that "Psychologically unbalanced phenomenon, will inspire individuals to restore the balance between them and the environment". Those who enter a completely different cultural environment will face the role conversion process. This conversion caused by psychological imbalance makes individuals need to be adjusted in order to rebalance the state.

Sam and Berry (2010) believe that the cultural adaptation process will be a cultural and psychological change. On the cultural level, the individual's collective activities and social customs will change. On the psychological level, the daily behavior criterion and the stress experience. In the process, the individual usually takes integration, assimilation, separation or marginalization coping strategies.

Previous cross-cultural adaptation studies based on individuals unable (or do not want) to leave the environment as a basic condition. However, in the present study, individuals (employees) able to leave the cultural environment (working environment of foreign enterprise) at any time, this cultural environment is also a non local cultural environment. Therefore, once the individuals (employees) are not adapted to the cultural environment (working environment in foreign enterprise), the individuals (employees) may have thought of leaving the environment and then generate turnover intention.

\subsection{The Concept of Turnover Intention}

Porter and Steers (1973) represents "turnover intention" is the next withdrawal behavior after employees experience dissatisfied. Mobley (1997) believe that the next withdrawal behavior after employees experience dissatisfied is "Thinking of quitting". Turnover intention is the last step before the turnover behavior after several step (thinking of quitting, looking for new job, comparison to other job opportunities).

Mobley el al. (1978) believe that turnover intention is the comprehensive reflect of job dissatisfaction, thinking of quitting, the tendency to looking for other job and other job possibilities. This is also the definition of this study.

Price's (2001) turnover process model has better predictive capability which examines the impact of environment, individual, structural and process variable on turnover. Price's turnover theory has the additive property, empirical Research added more variables. Price (2001) noted that further research should be strengthened in the intermediate process; pay more attention to the value of the adjustment function and some of the possible interaction between the variables.

Alfonso el al. (2004) research showed that there are differences in turnover factors and turnover process of employees those from different regions, different industries, different enterprise nature and different cultural background. However, the reason for this difference is not yet produced in-depth study.

Previous turnover intention studies based on employees and organization are from the same cultural background. However, we know that there are differences in turnover factors and turnover process of employees those from different cultural background. Therefore, if employees and organizations are from different cultural backgrounds, cultural factors should also affect turnover intention and behavior.

\section{Method}

According to the above reasoning, we try to prove if there is a causal relationship between turnover intention and cross-cultural adaptation and how it is affected. In order to better measuring, we will quantify the turnover intention and cross-cultural adaptation.

\subsection{Measurement Index Defining}

Cross cultural adaptation index is an indicator that reflects the adaptation ability of individual in the different cultural environment. Base on the result of cross-cultural adaptation questionnaire and principal component analysis; calculate the corresponding evaluation of individual cross-cultural adaptability. The final data of the cross culture adaptation questionnaire was analyzed by the principal component analysis method using SPSS software in order to calculate the individual's cross cultural adaptation ability evaluation results.

Turnover intention index is an indicator reflects the possibility of employee turnover. Base on the result of turnover intention questionnaire and principal component analysis; calculate the corresponding evaluation of individual turnover intention possibility. The final data of the turnover intention questionnaire was analyzed by the principal component analysis method using SPSS software in order to calculate the individual's turnover intention evaluation results. 


\subsection{Index Selection and Questionnaire Design}

\subsubsection{Cross Cultural Adaptation Index Selection and Questionnaire Design}

This paper selected six indicators to reflect the sample Cross cultural adaptation. Mastery of a foreign language has a certain impact on the cross-cultural adaptation, so questionnaire design also adding "Your mastery of a foreign language" and "your education level".

Designed five level variables, Such as 1:I did not feel; 2: General; 3: Okay; 4: Relatively strong; 5: Strong. Some indicators designed as below (full scale see Annex I)

A1: You like to make friends with foreigners.

A2: You can easily communicate with foreigners.

A3: You can easily make friends with foreigners.

A4: You feel excluded by foreigners.

A5: You can stand on their (foreigners) perspective on the issues.

$\begin{array}{lllll}1 & 2 & 3 & 4 & 5 \\ 1 & 2 & 3 & 4 & 5 \\ 1 & 2 & 3 & 4 & 5 \\ 1 & 2 & 3 & 4 & 5 \\ 1 & 2 & 3 & 4 & 5 \\ 1 & 2 & 3 & 4 & 5\end{array}$

A6: You quite understand some acts of foreigners.

3.2.2 Turnover Intention Index Selection and Questionnaire Design

Huang Chun Sheng (2004) developed the scale of turnover intention:

1) I want to keep working in this company;

2) Sometimes you feel tired with your current job and want to change your job;

3) If you have suitable jobs I will accept;

4) I will leave the current company within a year.

Zhang Yue (2007) used two indicators to reflect turnover intention.

1) I will look for others job opportunities;

2) If you have suitable jobs I will accept.

Based on the above scales and considering to local employment situation, we put another question "If the salary is same with state-owned enterprises, you will leave foreign company": Number from 1 5 indicates the level or possibility of each question (1: Definitely not; 2: Low probably; 3: Probably; 4: High probably and 5: Definitely yes).

$\mathrm{C} 1$ : Sometimes you feel tired with your current job and want to change your job.

$\begin{array}{lllll}1 & 2 & 3 & 4 & 5 \\ 1 & 2 & 3 & 4 & 5 \\ 1 & 2 & 3 & 4 & 5 \\ 1 & 2 & 3 & 4 & 5 \\ 1 & 2 & 3 & 4 & 5 \\ 1 & 2 & 3 & 4 & 5\end{array}$

C2: I will look for others job opportunities.

C3: If you have suitable jobs I will accept.

C4: I will leave the current company within a year.

C5: I will leave the current company within 3 year.

C6: If the salary is same with state-owned enterprises, you will leave foreign company. $\begin{array}{llllll}1 & 2 & 3 & 4 & 5\end{array}$

\subsection{Investigation Process}

The subjects of this study are local employees (Laotian) who work for foreign enterprises in Laos (Included European, Japanese, Korean, Chinese, Southeast Asia ... etc.). 500 invited, 300 recruited samples, 248 valid samples ( $82.66 \%$ of valid data).

We target to local employees who work for foreign enterprises in Laos base on the relationship with several associations (Lao students Association in Japan, Lao student association in China, Lao student association in Australia ... etc.). We used online survey to collect sample for this study.

Used below screening questions, only those respondents who are full time worker and work for foreign enterprises able to access to the main survey.

S1. Please select your working status:
1) Full time worker;
2) Part time worker;
3) Others. 
Only those select option 1 "Full time worker" on S1 will able to continue the survey, all of other option will be terminated.

S2. Please select your company type:

1) Foreign company;

2) Local company;

3) Government units;

4) Others.

Only those select option 1 "Foreign company" on S2 will able to continue the survey, all of other option will be terminated.

\subsection{Scale Test}

To ensure the validity of both turnover intention and cross cultural adaptation scales we use, we use the SPSS software to perform the following validation.

Table 1 presents the result of cross cultural adaptation scale test obtained. Cronbach's Alpha index is 0.811 which greater than 0.7 . Description the scales has good reliability.

Table 1. Reliability statistics

\begin{tabular}{cc}
\hline Cronbach's Alpha & N of Items \\
\hline 0.811 & 20 \\
\hline
\end{tabular}

Table 2 presents the result of turnover intention scale test obtained. We have 0.827 Cronbach's Alpha index which greater than 0.7 . Description the scales has good reliability.

Table 2. Reliability statistics

\begin{tabular}{cc}
\hline Cronbach's Alpha & N of Items \\
\hline 0.827 & 6 \\
\hline
\end{tabular}

\subsection{Analysis}

\subsubsection{Mean Analysis}

Table 3 presents the result of mean analysis of cross cultural adaptation, various factors concentrated in medium and medium high level. Middle high included "You can accept any change", "You like the challenge", "You like to make friends with foreigners", "You agree with the corporate culture in the organization you work for", "You can accept foreigners strange behavior", "You proficiency in a foreign language", "You like watching a foreign film", "You can easily make friends with foreigners", "You can understand their jokes and humor", "You can endure the harsh reality". The highest is "You can accept any change" and lowest is "You can endure the harsh reality".

Medium level included "You like to solve problems independently", "You think foreigners are friendly", "You quite understand some acts of foreigners", "If someone hurt you, you can accept his/her apology", "You can stand on their (foreigners) perspective on the issues", "You like foreign food", "You can accept a foreigner pursuit", "You can easily communicate with foreigners", "You feel excluded by foreigners". The highest is "You like to solve problems independently" and lowest is "You feel excluded by foreigners". 
Table 3. Descriptive statistics

\begin{tabular}{|c|c|c|c|}
\hline & $\mathrm{N}$ & Mean & Std. Deviation \\
\hline A1 & 248 & 3.217742 & 1.03 \\
\hline A2 & 248 & 2.524194 & 0.731 \\
\hline A3 & 248 & 3.03629 & 1.225 \\
\hline A4 & 248 & 2.169355 & 1.066 \\
\hline A5 & 248 & 2.895161 & 0.847 \\
\hline A6 & 248 & 2.967742 & 1.131 \\
\hline A7 & 248 & 2.971774 & 0.991 \\
\hline A8 & 248 & 3.16129 & 0.857 \\
\hline A9 & 248 & 3.189516 & 0.9 \\
\hline $\mathrm{A} 10$ & 248 & 2.850806 & 0.934 \\
\hline A11 & 248 & 2.810484 & 1.087 \\
\hline A12 & 248 & 3.016129 & 0.994 \\
\hline A13 & 248 & 3.080645 & 1.251 \\
\hline A14 & 248 & 3.112903 & 0.987 \\
\hline A15 & 248 & 3.096774 & 0.702 \\
\hline A16 & 248 & 3 & 1.006 \\
\hline A17 & 248 & 2.991935 & 0.969 \\
\hline A18 & 248 & 3.229839 & 1.145 \\
\hline A19 & 248 & 2.96371 & 0.991 \\
\hline $\mathrm{A} 20$ & 248 & 3.387097 & 1.085 \\
\hline Valid N (listwise) & 248 & & \\
\hline
\end{tabular}

Table 4 presents the result of mean analysis of turnover intention, various factors concentrated in medium and medium high level. Medium high included "I will leave the current company within 3 year" and "If you have suitable jobs I will accept", "I will leave the current company within 3 year" is highest.

Medium level included "If the salary is same with state-owned enterprises, you will leave foreign company.", "I will look for others job opportunities", "Sometimes you feel tired with your current job and want to change your job" and "I will leave the current company within a year". The highest is "If the salary is same with state-owned enterprises, you will leave foreign company." And lowest is "I will leave the current company within a year".

Table 4. Turnover intention descriptive statistics

\begin{tabular}{cccc}
\hline & $\mathrm{N}$ & Mean & Std. Deviation \\
\hline $\mathrm{C} 1$ & 248 & 2.58871 & 0.8 \\
$\mathrm{C} 2$ & 248 & 2.875 & 0.946 \\
$\mathrm{C} 3$ & 248 & 3 & 1.214 \\
$\mathrm{C} 4$ & 248 & 2.306452 & 1.139 \\
$\mathrm{C} 5$ & 248 & 3.193548 & 1.28 \\
C6 & 248 & 2.959677 & 1.21 \\
\hline
\end{tabular}

\subsubsection{Factor Analysis}

In order to calculate the cross cultural adaptation and turnover intention index, we first verify the following questionnaire data and pre calculation. 
Table 5 presents the result of KMO and Bartlett's Test on cross cultural adaptation scale obtained. KMO index is 0.682 which greater than 0.5 and Bartlett test value is 0.000 which less than 0.05 . Indicating that the scale has construct validity and suitable for factor analysis.

Table 5. KMO and Bartlett's Test

\begin{tabular}{lrr}
\hline Kaiser-Meyer-Olkin Measure of Sampling Adequacy. & .682 \\
\hline Bartlett's Test of Sphericity & Approx. Chi-Square & 1429.427 \\
& Df & 190 \\
& Sig. & .000 \\
\hline
\end{tabular}

Table 6 presents the result of total variance explained on cross cultural adaptation scale obtained.

Table 6. Total variance explained

\begin{tabular}{|c|c|c|c|c|c|c|c|c|c|}
\hline \multirow{2}{*}{ Component } & \multicolumn{3}{|c|}{ Initial Eigenvalues } & \multicolumn{3}{|c|}{ Extraction Sums of Squared Loadings } & \multicolumn{3}{|c|}{ Rotation Sums of Squared Loadings } \\
\hline & Total & $\%$ of Variance & Cumulative $\%$ & Total & $\%$ of Variance & Cumulative $\%$ & Total & $\%$ of Variance & Cumulative $\%$ \\
\hline 1 & 4.567 & 22.833 & 22.833 & 4.567 & 22.833 & 22.833 & 1.541 & 7.703 & 7.703 \\
\hline 2 & 1.968 & 9.842 & 32.675 & 1.968 & 9.842 & 32.675 & 1.483 & 7.416 & 15.119 \\
\hline 3 & 1.678 & 8.391 & 41.066 & 1.678 & 8.391 & 41.066 & 1.422 & 7.111 & 22.230 \\
\hline 4 & 1.483 & 7.417 & 48.483 & 1.483 & 7.417 & 48.483 & 1.383 & 6.914 & 29.143 \\
\hline 5 & 1.202 & 6.012 & 54.495 & 1.202 & 6.012 & 54.495 & 1.367 & 6.835 & 35.979 \\
\hline 6 & 1.153 & 5.764 & 60.259 & 1.153 & 5.764 & 60.259 & 1.343 & 6.714 & 42.693 \\
\hline 7 & 1.049 & 5.246 & 65.505 & 1.049 & 5.246 & 65.505 & 1.340 & 6.702 & 49.395 \\
\hline 8 & .900 & 4.502 & 70.007 & .900 & 4.502 & 70.007 & 1.265 & 6.325 & 55.720 \\
\hline 9 & .828 & 4.141 & 74.148 & .828 & 4.141 & 74.148 & 1.216 & 6.078 & 61.798 \\
\hline 10 & .768 & 3.839 & 77.988 & .768 & 3.839 & 77.988 & 1.201 & 6.003 & 67.801 \\
\hline 11 & .697 & 3.485 & 81.473 & .697 & 3.485 & 81.473 & 1.200 & 6.000 & 73.801 \\
\hline 12 & .672 & 3.360 & 84.833 & .672 & 3.360 & 84.833 & 1.142 & 5.708 & 79.509 \\
\hline 13 & .567 & 2.834 & 87.667 & .567 & 2.834 & 87.667 & 1.139 & 5.697 & 85.206 \\
\hline 14 & .519 & 2.593 & 90.259 & .519 & 2.593 & 90.259 & 1.011 & 5.053 & 90.259 \\
\hline 15 & .423 & 2.113 & 92.372 & & & & & & \\
\hline 16 & .403 & 2.017 & 94.389 & & & & & & \\
\hline 17 & .361 & 1.805 & 96.195 & & & & & & \\
\hline 18 & .278 & 1.391 & 97.585 & & & & & & \\
\hline 19 & .249 & 1.244 & 98.829 & & & & & & \\
\hline 20 & .234 & 1.171 & 100.000 & & & & & & \\
\hline
\end{tabular}

Based on the output of eigenvalues greater than 0.5. Using factor analysis, we got 14 factors from Lao employee cross cultural adaptation scale, cover $90 \%$ of all information. The third column shows the factor after rotation, total variance contribution rate unchanged, indicating that does not affect the original Common degree.

Table 7 presents the result of Component Score Coefficient Matrix on cross cultural adaptation scale obtained. 
Table 7. Component score coefficient matrix

\begin{tabular}{|c|c|c|c|c|c|c|c|c|c|c|c|c|c|c|}
\hline & \multicolumn{14}{|c|}{ Component } \\
\hline & 1 & 2 & 3 & 4 & 5 & 6 & 7 & 8 & 9 & 10 & 11 & 12 & 13 & 14 \\
\hline A1 & -.067 & .258 & -.067 & .389 & -.083 & .184 & .109 & -.165 & -.061 & -.163 & -.012 & -.067 & -.038 & .074 \\
\hline A2 & -.076 & .079 & .091 & -.065 & -.081 & -.058 & .004 & -.024 & -.085 & .878 & -.023 & -.133 & -.100 & .055 \\
\hline A3 & -.022 & .809 & -.008 & -.046 & -.080 & -.056 & -.203 & -.085 & -.051 & .129 & -.200 & -.048 & .003 & -.043 \\
\hline A4 & -.160 & .381 & .026 & -.263 & .031 & -.312 & .327 & .450 & .021 & -.144 & .260 & -.064 & -.052 & -.294 \\
\hline A5 & .106 & .093 & .437 & .080 & .322 & .041 & -.087 & -.334 & -.140 & -.078 & .032 & .196 & -.221 & -.091 \\
\hline A6 & -.029 & -.057 & -.069 & -.059 & .139 & -.036 & -.135 & .065 & -.034 & -.103 & .007 & .941 & -.006 & .016 \\
\hline A7 & -.128 & -.061 & -.071 & -.219 & -.003 & .740 & -.068 & .110 & .098 & -.002 & .042 & -.044 & .106 & -.013 \\
\hline A8 & -.024 & -.106 & -.059 & -.026 & .053 & .008 & .058 & -.071 & -.026 & .033 & .077 & .034 & -.025 & .882 \\
\hline A9 & .032 & -.180 & -.066 & .026 & -.035 & .125 & -.112 & -.094 & -.177 & .003 & .927 & -.008 & -.075 & .143 \\
\hline A10 & .003 & -.148 & .003 & -.070 & -.156 & -.066 & .844 & .008 & -.004 & .040 & -.084 & -.123 & .075 & .100 \\
\hline A11 & .702 & -.027 & .092 & -.118 & -.032 & -.241 & -.021 & -.129 & .028 & -.039 & -.027 & -.003 & .248 & -.062 \\
\hline A12 & -.138 & -.083 & .083 & -.086 & .761 & .030 & -.127 & -.032 & .014 & -.119 & -.124 & .049 & -.064 & .210 \\
\hline A13 & -.124 & -.218 & .153 & .280 & -.263 & -.069 & .222 & -.083 & .559 & -.014 & -.139 & .216 & .086 & -.156 \\
\hline A14 & .063 & -.062 & -.351 & -.023 & .437 & -.219 & .104 & .028 & .128 & .302 & .236 & .082 & .036 & -.483 \\
\hline A15 & .479 & -.164 & -.052 & .256 & -.344 & .413 & .068 & .079 & -.121 & -.090 & -.053 & -.056 & -.164 & .141 \\
\hline A16 & .011 & -.020 & -.112 & .729 & -.021 & -.146 & -.117 & .122 & -.178 & .030 & .002 & -.081 & -.022 & -.028 \\
\hline A17 & .157 & -.053 & -.093 & -.010 & -.134 & .077 & .018 & -.009 & -.149 & -.095 & -.076 & -.027 & .977 & .021 \\
\hline A18 & .013 & -.024 & .688 & -.162 & -.037 & -.091 & .001 & .079 & .011 & .111 & -.036 & -.130 & -.012 & -.039 \\
\hline A19 & .021 & .021 & -.020 & -.180 & .087 & .136 & -.143 & -.065 & .823 & -.076 & -.115 & -.093 & -.160 & .098 \\
\hline A20 & -.031 & -.129 & -.031 & .090 & -.077 & .136 & -.082 & .758 & -.071 & -.021 & -.112 & .084 & -.014 & .014 \\
\hline
\end{tabular}

Table 8. KMO and Bartlett's test

\begin{tabular}{llr}
\hline Kaiser-Meyer-Olkin Measure of Sampling Adequacy. & .819 \\
\hline Bartlett's Test of Sphericity & Approx. Chi-Square & 525.218 \\
& Df & 15 \\
& Sig. & .000 \\
\hline
\end{tabular}

Table 8 presents the result of KMO and Bartlett's Test on turnover intention scale obtained. KMO index is 0.819 which greater than 0.5 and Bartlett test value is 0.000 which less than 0.05 . Indicating that the scale has construct validity and suitable for factor analysis.

Table 9 presents the result of total variance explained on turnover intention scale obtained.

Table 9. Total variance explained

\begin{tabular}{|c|c|c|c|c|c|c|c|c|c|}
\hline \multirow{2}{*}{ Component } & \multicolumn{3}{|c|}{ Initial Eigenvalues } & \multicolumn{3}{|c|}{ Extraction Sums of Squared Loadings } & \multicolumn{3}{|c|}{ Rotation Sums of Squared Loadings } \\
\hline & Total & $\%$ of Variance & Cumulative $\%$ & Total & $\%$ of Variance & Cumulative $\%$ & Total & $\%$ of Variance & Cumulative $\%$ \\
\hline 1 & 3.294 & 54.908 & 54.908 & 3.294 & 54.908 & 54.908 & 1.739 & 28.98 & 28.98 \\
\hline 2 & 0.768 & 12.798 & 67.706 & 0.768 & 12.798 & 67.706 & 1.432 & 23.864 & 52.844 \\
\hline 3 & 0.708 & 11.804 & 79.511 & 0.708 & 11.804 & 79.511 & 1.072 & 17.861 & 70.705 \\
\hline 4 & 0.513 & 8.544 & 88.055 & 0.513 & 8.544 & 88.055 & 1.041 & 17.35 & 88.055 \\
\hline 5 & 0.4 & 6.667 & 94.722 & & & & & & \\
\hline 6 & 0.317 & 5.278 & 100 & & & & & & \\
\hline
\end{tabular}


Based on the output of eigenvalues greater than 0.5. Using factor analysis, we got 4 factors from Lao employee turnover intention scale, cover $88.055 \%$ of all information. The third column shows the factor after rotation, total variance contribution rate unchanged, indicating that does not affect the original Common degree.

Table 10 presents the result of Component Score Coefficient Matrix on turnover intention scale obtained.

Table 10. Component score coefficient matrix

\begin{tabular}{ccccc}
\hline & \multicolumn{3}{c}{ Component } \\
\cline { 2 - 5 } & 1 & 2 & 3 & 4 \\
\hline C1 & 0.091 & 0.526 & -0.439 & 0.12 \\
C2 & -0.291 & 0.867 & 0.173 & -0.309 \\
C3 & -0.191 & -0.225 & -0.067 & 1.158 \\
C4 & 0.585 & -0.005 & 0.081 & -0.353 \\
C5 & 0.669 & -0.308 & -0.121 & 0.055 \\
C6 & -0.092 & -0.136 & 1.005 & -0.102 \\
\hline
\end{tabular}

\subsection{Index Calculation}

Base on above pre calculated of both cross cultural adaptation and turnover intention, we have both index calculation processes below:

\subsubsection{Cross Cultural Adaptation Index}

To get the cross cultural adaptation index of Lao employee who work in foreign enterprises. We need to calculate the standardization scores of each factor using below formula:

$$
Y=(X-u) / \sigma
$$

$\mathrm{X}$ is the variable value, $\mathrm{u}$ is the sample mean, $\sigma$ is the sample standard deviation. Therefore, standardized scores GI = Score Coefficient ${ }_{11} *\left(A_{11}-u_{11}\right) / \sigma 1_{1}+$ Score Coefficient ${ }_{21} *\left(A_{21}-u_{21}\right) / \sigma_{2}+\ldots+$ Score Coefficient $201 *\left(A_{20}-u_{20}\right) / \sigma_{20}$

Base on Table 3 and Table7. Case in D1, we got factor standardization score of D1 is:

$\mathrm{G} 1=\{[(4-3.217742) / 1.030392] *(-0.066987)\}+\{[(3-2.524194) / 0.730633] *(-0.076188)\}+\{[(2-3.036290) / 1.225031$ ]$\left.^{*}(-0.022397)\right\}+\{[(2-2.169355) / 1.066393] *(-0.160206)\}+\{[(4-2.895161) / 0.847177] *(0.105668)\}+\{[(3-2.96774$ $2) / 1.130623] *(-0.028686)\}+\{[(3-2.971774) / 0.991466] *(-0.128361)\}+\{[(4-3.161290) / 0.857329] *(-0.024178)\}+$ $\{[(4-3.189516) / 0.900051] *(0.031775)\}+\{[(3-2.850806) / 0.934017] *(0.002799)\}+\{[(3-2.810484) / 1.087457] *(0.7$ $02463)\}+\{[(3-3.016129) / 0.993777] *(-0.138336)\}+\{[(4-3.080645) / 1.250728] *(-0.124306)\}+\{[(4-3.112903) / 0.9$ $\left.87449]^{*}(0.063407)\right\}+\left\{[(5-3.096774) / 0.701870]^{*}(0.479365)\right\}+\{[(4-3.000000) / 1.006055] *(0.010631)\}+\{[(3-2.99$ $1935) / 0.969126] *(0.157013)\}+\{[(4-3.229839) / 1.145134] *(0.013423)\}+\{[(3-2.963710) / 0.991203] *(0.020857)\}$ $+\left\{[(4-3.387097) / 1.085120]^{*}(-0.031404)\right\}=1.476712$

Similarly, we obtain:

$\mathrm{G} 2=-1.440320 ; \mathrm{G} 3=0.463272 ; \mathrm{G} 4=1.933737 ; \mathrm{G} 5=-0.471823 ; \mathrm{G} 6=1.087744 ; \mathrm{G} 7=0.448031 ; \mathrm{G} 8=0.031734 ; \mathrm{G} 9=-0.40$ $4987 ; \mathrm{G} 10=0.399926 ; \mathrm{G} 11=0.913569 ; \mathrm{G} 12=0.153286 ; \mathrm{G} 13=-0.791976 ; \mathrm{G} 14=0.839985$

Then, calculate cross cultural adaptation index according to Table 6. Case in D1, we got cross cultural adaptation index is:

$\mathrm{L} 1=(1.476712 * 0.077028)+(-1.440320 * 0.074158)+(0.463272 * 0.071112)+(1.933737 * 0.069138)+(-0.471823 * 0.0$ $68354)+(1.087744 * 0.067137)+(0.448031 * 0.067024)+(0.031734 * 0.063250)+(-0.404987 * 0.060777)+(0.399926 *$ $0.060030)+(0.913569 * 0.059998)+(0.153286 * 0.057081)+(-0.791976 * 0.056973)+(0.839985 * 0.050534)=$ 0.306669

Similarly, we can calculate D2 to D248's cross cultural adaptation index.

3.6.2 Turnover Intention Index

To get the turnover intention index of Lao employee who work in foreign enterprises. We need to calculate the standardization scores of each factor using below formula:

$$
Y=(C-u) / \sigma
$$


$\mathrm{C}$ is the variable value, $\mathrm{u}$ is the sample mean, $\sigma$ is the sample standard deviation. Therefore, standardized scores $H I=$ Score Coefficient ${ }_{11} *\left(C_{1}-u_{1}\right) / \sigma_{1}+$ Score Coefficient ${ }_{21} *\left(C_{2}-u_{2}\right) / \sigma_{2}+\ldots \ldots .+$ Score Coefficient ${ }_{61} *\left(C_{6}-u_{6}\right) / \sigma_{6}$.

Base on Table 4 and Table10. Case in D1, we got factor standardization score of D1 is:

$\mathrm{H} 1=\left\{[(1-2.588710) / 0.799920]^{*}(0.091475)\right\}+\{[(2-2.875000) / 0.946173] *(-0.290968)\}+\{[(1-3.000000) / 1.213954]$ $*(-0.191334)\}+\{[(2-2.306452) / 1.139482] *(0.585249)\}+\{[(1-3.193548) / 1.280039] *(0.669440)\}+\{[(1-2.959677)$ $\left./ 1.209940]^{*}(-0.091592)\right\}=-0.753612$

Similarly, we obtain $\mathrm{H} 2=-0.726112 ; \mathrm{H} 3=-0.620500$ and $\mathrm{H} 4=-1.695121$.

Then, calculate turnover intention index according to Table 9. Case in D1, we got cross cultural adaptation index is:

$M 1=(-0.753612 * 0.289799)+(-0.726112 * 0.238644)+(-0.620500 * 0.178607)+(-1.695121 * 0.173499)=-0.796605$

Similarly, we can calculate D2 to D248's turnover intention index.

According to above method, we got cross cultural adaptation index and turnover intention index of each respondent on Table 10 (full content see Annex I I).

Table 10. Cross cultural adaptation index, turnover intention index (partial content)

\begin{tabular}{ccc}
\hline Respondents & Cross cultural adaptation index & Turnover intention index \\
\hline D1 & 0.306669 & -0.796605 \\
D2 & 0.021867 & -0.123961 \\
D3 & -0.117529 & 0.166742 \\
D4 & 0.116911 & -0.038063 \\
D5 & -0.312975 & 0.762096 \\
D6 & 0.001992 & -0.273485 \\
D7 & -0.474553 & 0.719962 \\
D8 & 0.030495 & -0.103714 \\
D9 & -0.074048 & -0.325765 \\
\hline
\end{tabular}

\section{Results}

Base on above calculated result of both cross cultural adaptation and turnover intention index. Using SPSS software for correlation analysis and regression as follows to find out whether there is linear correlation and regression model between both indexes.

\subsection{Correlation Analysis}

Figure 1 presents the result of correlation analysis between cross cultural adaptation index and turnover intention index obtained. Base on Table 10, using SPSS "Scatter" module to find out if there is a linear relationship between cross cultural adaptation index and turnover intention index.

Figure 1 result showed that there is a strong negative correlation between cross cultural adaptation index and turnover intention index. 


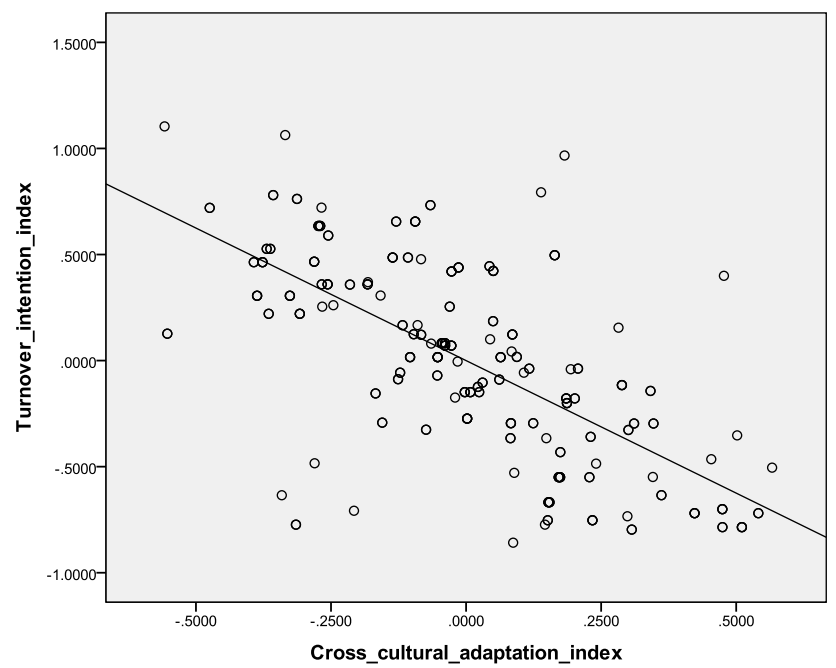

Figure 1. Relationship between cross cultural adaptation index and turnover intention index

Table 11, Table 12 and Table 13 presents the result of a linear regression between cross cultural adaptation index and turnover intention index obtained.

Table 11. Model summary ${ }^{\mathrm{b}}$

\begin{tabular}{cccccc}
\hline Model & R & R Square & Adjusted R Square & Std. Error of the Estimate & Durbin-Watson \\
\hline 1 & $.673^{\mathrm{a}}$ & 0.453 & 0.451 & 0.3338 & 2.295 \\
\hline
\end{tabular}

a Predictors: (Constant), Cross cultural adaptation index;

b. Dependent Variable: Turnover intention index.

Table 12. ANOVA ${ }^{\mathrm{b}}$

\begin{tabular}{ccccccc}
\hline & Model & Sum of Squares & df & Mean Square & F & Sig. \\
\hline 1 & Regression & 22.71 & 1 & 22.71 & 203.782 & $.000^{\mathrm{a}}$ \\
& Residual & 27.415 & 246 & 0.111 & & \\
& Total & 50.125 & 247 & & &
\end{tabular}

a. Predictors: (Constant), Cross cultural adaptation index;

b. Dependent Variable: Turnover intention index.

Table 13. Coefficients ${ }^{\mathrm{a}}$

\begin{tabular}{|c|c|c|c|c|c|c|}
\hline & \multirow{2}{*}{ Model } & \multicolumn{2}{|c|}{ Unstandardized Coefficients } & \multirow{2}{*}{$\begin{array}{c}\text { Standardized Coefficients } \\
\text { Beta }\end{array}$} & \multirow{2}{*}{$\mathrm{t}$} & \multirow{2}{*}{ Sig. } \\
\hline & & B & Std. Error & & & \\
\hline \multirow{2}{*}{1} & (Constant) & $3.45 \mathrm{E}-07$ & 0.021 & & 0 & 1 \\
\hline & Cross cultural adaptation index & -1.249 & 0.088 & -0.673 & -14.3 & 0 \\
\hline
\end{tabular}

a. Dependent Variable: Turnover intention index.

Base on the result of a linear regression between cross cultural adaptation index and turnover intention index obtained. Cross cultural adaptation index each increase of 1 unit, turnover intention index will be reduced for 1.249 units.

F test: Base on Table 12. We got $F$ test significant value is 0 which less than 0.05 , description that our regression 
model has statistical significance, regression results is significant.

$T$ test: Base on Table 13. Sig. of dependent variable (Cross cultural adaptation index) is 0 which less than 0.05 , description that the interpretation of cross cultural adaptation index to turnover intention index has statistical significance, the correlation of both index is significant.

Correlation coefficient: Base on Table 11. The correlation coefficient of cross cultural adaptation index and turnover intention index is 0.673 , Durbin-Wastson index is 2.295 , linear correlation between both indexes is significant.

Base on above. We found that there is linear correlation between cross cultural adaptation index and turnover intention index and there is a strong negative correlation. For the target people of this study, cross cultural adaptation is an important factor of turnover intention.

\section{Discussion and Conclusion}

\subsection{Theoretical and Practical Implications}

Previous studies about turnover issue focused on job satisfaction, organizational commitment, organizational support...etc. Moreover, most of previous researches based on the employee and the organization are belong to the same cultural background.

The results of this study show that there is linear correlation between cross cultural adaptation index and turnover intention index and there is a strong negative correlation. This means that the cultural factors can directly affect to the turnover issue of Lao employees who work in foreign enterprise. Cross cultural adaptation is not a small obstacle but it's one of the most important factors which is able to determine employees to leave or stay, especially for employees and organization are from different cultural background.

Working environment in foreign enterprises those running business in Laos is rather special, especially cultural environment which influenced by foreign enterprises their own enterprise culture, native culture and local culture, such culture even different from any single culture.

Since employee belongs to local culture background, which has different working culture environment; such differences included information handling, information understanding level and mindset. Under a particular traditional culture and living environment, people have common distinctive features. In order to properly train employees, improve their own efficiency and reduce the hidden costs by turnover; Enterprises should have an open mind and good learning ability. Enterprises not only need to understand the performance characteristics of its distinctive shape, more is needed to understand there are a lot of factors behind; contact between them and its impact.

We can reduce turnover intention by increasing cross cultural adaptation. However, cross-cultural adaptation was the result of employees and businesses from different cultural backgrounds, this issue is generated after employees joined foreign enterprise. Therefore, we know the following:

(1) Cross cultural adaptation issue is the result of the mutual influence of both employees and foreign enterprise;

(2) Babiker et al. (1980) proposed that the psychological and cultural adaptation of the problem is precisely because of the differences between home culture and the new culture. Those who feel a greater cultural difference will be faced with a greater change in life in the process of cross-cultural adaptation, which will give them more stress, depression and other adaptation problems. According to Babiker's theory, we predict that Employees work in different types of foreign enterprise (For example: European enterprise, American enterprise, Japanese enterprise, Korean enterprise, Chinese enterprise, etc.) will have different level of cross cultural adaptation issue; (3) While the cultural environment of foreign enterprise impact to the local employees, the local employees also impact on the cultural environment of foreign enterprise. Therefore, in order to reduce cross cultural adaptation issue, foreign enterprise should establish an effective way to communicate and cultural exchange mechanism.

\subsection{Limitations}

As in any study, there are some limitations in our study as well. Definitely, the larger sample size would enable us to attain more generalizable results. Secondly, using convenience sampling method, our sample may have some deficiencies in terms of variability and representability of the population.

\subsection{Suggestions}

We empirically articulate the manifestation of cross-cultural adaptation as a variable. Yet, it may have some other systematic influences on the above-posited relationships. First, we suggest researchers consider a moderated 
mediation effect into their research based on modeling. Second, it is better to replicate this research with a larger sample size in order to validate our results. Third, in future, the role of other individual factors such as organization type and personality traits on cross cultural adaptation can be investigated.

\section{References}

Alfonso, Sousa-Poza, Fred, Henneberger. (2004). Analyzing job mobility with job turnover intentions: An international comparative Study. Journal of Economic Issues, 38(1), 113-25.

Babiker, I. E., Cox, J. L., \& Miller, P. (1980). The measurement of cultural distance and its relationship to medical consultations, symptomatology, and examination performance of overseas students at Edinburgh University. Social Psychiatry, 15(3), 109-116. http://dx.doi.org/10.1007/BF00578141

Chun-Sheng, H. (2004). The relationship between job satisfaction, organization commitment and turnover intention (Unpublished Manuscript). Amoy University.

Lin-Gang, Z., \& Jian-Hua, F. (2005). Social support theory: Literature review. Journal of Guang Xi University, 26(7), 11-14.

Mobley, E. H., Horner, S. O., Hollingsworth, A. T. (1978). An evaluation of precursors of hospital employee turnover. Journal of Applied Psychology, 63, 408-414. http://dx.doi.org/10.1037/0021-9010.63.4.408

Mobley, W. H. (1977). Intermediate linkages in the relationship between job satisfaction and employee turnover. Journal of Applied Psychology, 62(1), 237-240. http://dx.doi.org/10.1037/0021-9010.62.2.237

Porter, L. W., \& Steers, R. M. (1973). Organizational, work and personal factors in employee turnover and absenteeism. Psychological Bulletin, 80, 151-176. http://dx.doi.org/10.1037/h0034829

Price. (2001). Reflections on determinants of voluntary turnover. International Journal of Manpower, 22, 600-624. http://dx.doi.org/10.1108/EUM0000000006233

Qing, Z. (2010). Adaptation structure, influence factors and relationship with job satisfaction: A study employees Shanghai those return from overseas (Unpublished Manuscript). East China Normal University.

Redfield, R., Linton, R., \& Herskovits, M. J. (1936). Memorandum on the study of acculturation. American Anthropologist, 38(2), 149-152. http://dx.doi.org/10.1525/aa.1936.38.1.02a00330

Sam, D. L., \& Berry, J. W. (2010). Acculturation: When individuals and groups of different cultural backgrounds meet. Perspectives on Psychological Science, 5(4), 472-481. http://dx.doi.org/10.1177/1745691610373075

Wei, Y., \& Gang, Z. (2005). Cross cultural psychology: Cross cultrual adaptation. Advances in Psychological Science, 6, 134-144.

Ying, G. (1997). The research progress of cross cultural psychology. Journal of Sichuan Normal University, 4 , 90-95.

Yue, Z. (2007). Empirical research of organization commitment and turnover intention (Unpublished Manuscript). Southwestern University of Finance and Economics.

\section{Appendix A}

\section{Questionnaire}

Thank you very much for participation on this survey.

This is an academic study which discuss about cross cultural adaptation and turnover intention. We try to understand if cross cultural adaptation will effect to the turnover intention.

We would like to know how you feel about each part of cross cultural adaptation and your possibility to leave your current company.

There is no correct or incorrect option in this survey. Please select the option which best descript to your current situation.

Your provided information will not be disclose to any third part. All information will be use on unified analysis.

S1 : Please select your working status

1. Full time worker Continue

2. Part time worker Terminated 
S2. Please select your company type

\begin{tabular}{lcl}
\hline 1. & Foreign company & Continue \\
2. & Local company & Terminated \\
3. & Government units & Terminated \\
4. & Others & Terminated \\
\hline
\end{tabular}

Part 1: Cross cultural adaptation

Below questions asking about the ability of your cross cultural adaptation. Please view each question carefully and select the option which best descript to your current situation.

\begin{tabular}{|c|c|c|c|c|c|}
\hline & $\begin{array}{l}\text { I did not } \\
\text { feel }\end{array}$ & General & Okay & $\begin{array}{l}\text { Relatively } \\
\text { strong }\end{array}$ & Strong \\
\hline A1: You like to make friends with foreigners & 1 & 2 & 3 & 4 & 5 \\
\hline A2 : You can easily communicate with foreigners & 1 & 2 & 3 & 4 & 5 \\
\hline A3 : You can easily make friends with foreigners & 1 & 2 & 3 & 4 & 5 \\
\hline A4 : You feel excluded by foreigners & 1 & 2 & 3 & 4 & 5 \\
\hline A5 : You can stand on their (foreigners) perspective on the issues & 1 & 2 & 3 & 4 & 5 \\
\hline A6: You quite understand some acts of foreigners & 1 & 2 & 3 & 4 & 5 \\
\hline A7 : You think foreigners are friendly & 1 & 2 & 3 & 4 & 5 \\
\hline A8 : You can accept foreigners strange behavior & 1 & 2 & 3 & 4 & 5 \\
\hline A9 : You agree with the corporate culture in the organization you work for & 1 & 2 & 3 & 4 & 5 \\
\hline A10 : You like foreign food & 1 & 2 & 3 & 4 & 5 \\
\hline A11 : You can accept a foreigner pursuit & 1 & 2 & 3 & 4 & 5 \\
\hline A12 : You can understand their jokes and humor & 1 & 2 & 3 & 4 & 5 \\
\hline A13 : You like watching a foreign film & 1 & 2 & 3 & 4 & 5 \\
\hline A14 : You proficiency in a foreign language & 1 & 2 & 3 & 4 & 5 \\
\hline $\begin{array}{l}\text { A15 : Your education level (1: Middle school, 2: High school, 3: } \\
\text { Undergraduate, 4: Master, 5: PHD) }\end{array}$ & 1 & 2 & 3 & 4 & 5 \\
\hline A16 : You can endure the harsh reality & 1 & 2 & 3 & 4 & 5 \\
\hline A17 : You like to solve problems independently & 1 & 2 & 3 & 4 & 5 \\
\hline A18 : You like the challenge & 1 & 2 & 3 & 4 & 5 \\
\hline A19 : If some one hurt you, you can accept his/her apology & 1 & 2 & 3 & 4 & 5 \\
\hline A20 : You can accept any change & 1 & 2 & 3 & 4 & 5 \\
\hline
\end{tabular}

Part 2: Turnover intention

Below questions asking about your possibility to leave your current company.Please view each question carefully and select the option which best descript to your current situation.

\begin{tabular}{|c|c|c|c|c|c|}
\hline & $\begin{array}{c}\text { Definitely } \\
\text { not }\end{array}$ & $\begin{array}{c}\text { Low } \\
\text { probably }\end{array}$ & Probably & High probably & $\begin{array}{c}\text { Definitely } \\
\text { yes }\end{array}$ \\
\hline $\begin{array}{l}\mathrm{C} 1: \text { Sometimes you feel tired with your current job and want to change } \\
\text { your job }\end{array}$ & 1 & 2 & 3 & 4 & 5 \\
\hline C2 : I will look for others job opportunities & 1 & 2 & 3 & 4 & 5 \\
\hline C3 : If you have suitable jobs I will accept & 1 & 2 & 3 & 4 & 5 \\
\hline $\mathrm{C} 4$ : I will leave the current company within a year & 1 & 2 & 3 & 4 & 5 \\
\hline C5: I will leave the current company within 3 year & 1 & 2 & 3 & 4 & 5 \\
\hline $\begin{array}{l}\text { C6 : If the salary is same with state-owned enterprises, you will leave } \\
\text { foreign company. }\end{array}$ & 1 & 2 & 3 & 4 & 5 \\
\hline
\end{tabular}




\section{Appendix B}

Respondent Cross cultural adaptation index Turnover intention index Respondent Cross cultural adaptation index Turnover intention index

\begin{tabular}{|c|c|c|c|c|c|}
\hline D1 & 0.306669 & -0.796605 & D125 & 0.345718 & -0.548651 \\
\hline D2 & 0.021867 & -0.123961 & D126 & 0.501781 & -0.352595 \\
\hline D3 & -0.117529 & 0.166742 & D127 & 0.29865 & -0.733838 \\
\hline D4 & 0.116911 & -0.038063 & D128 & -0.015668 & -0.004543 \\
\hline D5 & -0.312975 & 0.762096 & D129 & 0.146033 & -0.77335 \\
\hline D6 & 0.001992 & -0.273485 & D130 & 0.087109 & -0.858353 \\
\hline D7 & -0.474553 & 0.719962 & D131 & -0.334792 & 1.063066 \\
\hline D8 & 0.030495 & -0.103714 & D132 & -0.083362 & 0.477843 \\
\hline D9 & -0.074048 & -0.325765 & D133 & -0.089777 & 0.167125 \\
\hline D10 & -0.255115 & 0.589558 & D134 & 0.044473 & 0.100437 \\
\hline D11 & 0.230585 & -0.359382 & D135 & -0.158263 & 0.306676 \\
\hline D12 & -0.155178 & -0.292479 & D136 & 0.089152 & -0.529153 \\
\hline D13 & -0.043207 & 0.081856 & D137 & -0.266246 & 0.254277 \\
\hline D14 & -0.065981 & 0.732616 & D138 & 0.453837 & -0.464897 \\
\hline D15 & 0.207167 & -0.037676 & D139 & 0.182245 & 0.966663 \\
\hline D16 & 0.082206 & -0.365846 & D140 & -0.207469 & -0.707818 \\
\hline D17 & -0.053262 & -0.07031 & D141 & 0.107003 & -0.057059 \\
\hline D18 & -0.167568 & -0.154814 & D142 & -0.280357 & -0.484016 \\
\hline D19 & 0.163993 & 0.496694 & D143 & -0.341086 & -0.63467 \\
\hline D20 & -0.552984 & 0.126879 & D144 & 0.193753 & -0.040828 \\
\hline D21 & 0.341387 & -0.143078 & D145 & 0.566463 & -0.504648 \\
\hline D22 & 0.174475 & -0.431616 & D146 & 0.240662 & -0.485767 \\
\hline D23 & 0.049966 & 0.185743 & D147 & 0.138782 & 0.79333 \\
\hline D24 & 0.361638 & -0.634549 & D148 & 0.477246 & 0.399657 \\
\hline D25 & -0.12208 & -0.056939 & D149 & -0.064635 & 0.080107 \\
\hline D26 & 0.001992 & -0.273485 & D150 & -0.245679 & 0.260976 \\
\hline D27 & 0.228307 & -0.549783 & D151 & -0.181669 & 0.369678 \\
\hline D28 & 0.093898 & 0.017221 & D152 & 0.148515 & -0.365846 \\
\hline D29 & -0.214984 & 0.358276 & D153 & 0.282155 & 0.155107 \\
\hline D30 & -0.314905 & -0.77335 & D154 & -0.020348 & -0.174312 \\
\hline D31 & 0.300426 & -0.327015 & D155 & -0.26764 & 0.721096 \\
\hline D32 & -0.357039 & 0.779959 & D156 & 0.084278 & 0.042917 \\
\hline D33 & -0.362355 & 0.526679 & D157 & 0.163993 & 0.496694 \\
\hline D34 & -0.096896 & 0.124226 & D158 & -0.55809 & 1.103947 \\
\hline D35 & 0.31093 & -0.296621 & D159 & 0.306669 & -0.796605 \\
\hline D36 & -0.125883 & -0.087797 & D160 & 0.021867 & -0.123961 \\
\hline D37 & 0.15123 & -0.752716 & D161 & -0.117529 & 0.166742 \\
\hline D38 & 0.061504 & -0.089545 & D162 & 0.116911 & -0.038063 \\
\hline D39 & -0.392923 & 0.463675 & D163 & -0.312975 & 0.762096 \\
\hline D40 & 0.540974 & -0.719435 & D164 & 0.001992 & -0.273485 \\
\hline
\end{tabular}




\begin{tabular}{|c|c|c|c|c|c|}
\hline D41 & -0.107557 & 0.485793 & D165 & -0.474553 & 0.719962 \\
\hline D42 & 0.124239 & -0.295247 & D166 & 0.030495 & -0.103714 \\
\hline D43 & -0.365334 & 0.220657 & D167 & -0.074048 & -0.325765 \\
\hline D44 & -0.045257 & 0.081976 & D168 & -0.255115 & 0.589558 \\
\hline D45 & 0.201211 & -0.178454 & D169 & 0.230585 & -0.359382 \\
\hline D46 & -0.030273 & 0.254277 & D170 & -0.155178 & -0.292479 \\
\hline D47 & 0.154511 & -0.667953 & D171 & -0.043207 & 0.081856 \\
\hline D48 & -0.052644 & 0.016207 & D172 & -0.065981 & 0.732616 \\
\hline D49 & -0.267096 & 0.359409 & D173 & 0.207167 & -0.037676 \\
\hline D50 & 0.474524 & -0.785205 & D174 & 0.082206 & -0.365846 \\
\hline D51 & -0.129397 & 0.655329 & D175 & -0.053262 & -0.07031 \\
\hline D52 & 0.024233 & -0.148734 & D176 & -0.167568 & -0.154814 \\
\hline D53 & -0.369053 & 0.526679 & D177 & 0.163993 & 0.496694 \\
\hline D54 & -0.083173 & 0.121723 & D178 & -0.552984 & 0.126879 \\
\hline D55 & 0.346901 & -0.296621 & D179 & 0.341387 & -0.143078 \\
\hline D56 & 0.043303 & 0.444678 & D180 & 0.174475 & -0.431616 \\
\hline D57 & 0.233911 & -0.752716 & D181 & 0.049966 & 0.185743 \\
\hline D58 & 0.063935 & 0.015968 & D182 & 0.361638 & -0.634549 \\
\hline D59 & -0.376761 & 0.463675 & D183 & -0.12208 & -0.056939 \\
\hline D60 & 0.422932 & -0.719435 & D184 & 0.001992 & -0.273485 \\
\hline D61 & -0.135914 & 0.485793 & D185 & 0.228307 & -0.549783 \\
\hline D62 & 0.082961 & -0.295247 & D186 & 0.093898 & 0.017221 \\
\hline D63 & -0.30777 & 0.220657 & D187 & -0.214984 & 0.358276 \\
\hline D64 & -0.038983 & 0.081976 & D188 & -0.314905 & -0.77335 \\
\hline D65 & 0.185409 & -0.178454 & D189 & 0.300426 & -0.327015 \\
\hline D66 & 0.050333 & 0.422677 & D190 & -0.357039 & 0.779959 \\
\hline D67 & 0.171428 & -0.549783 & D191 & -0.362355 & 0.526679 \\
\hline D68 & -0.052644 & 0.016207 & D192 & -0.096896 & 0.124226 \\
\hline D69 & -0.256243 & 0.359409 & D193 & 0.31093 & -0.296621 \\
\hline D70 & 0.510496 & -0.785205 & D194 & -0.125883 & -0.087797 \\
\hline D71 & -0.094154 & 0.655329 & D195 & 0.15123 & -0.752716 \\
\hline D72 & -0.002407 & -0.148734 & D196 & 0.061504 & -0.089545 \\
\hline D73 & -0.326118 & 0.305541 & D197 & -0.392923 & 0.463675 \\
\hline D74 & -0.03887 & 0.070575 & D198 & 0.540974 & -0.719435 \\
\hline D75 & 0.1867 & -0.200452 & D199 & -0.107557 & 0.485793 \\
\hline D76 & -0.013968 & 0.438902 & D200 & 0.124239 & -0.295247 \\
\hline D77 & 0.152455 & -0.667953 & D201 & -0.365334 & 0.220657 \\
\hline D78 & -0.103693 & 0.016207 & D202 & -0.045257 & 0.081976 \\
\hline D79 & -0.281088 & 0.466178 & D203 & 0.201211 & -0.178454 \\
\hline D80 & 0.474341 & -0.700436 & D204 & -0.030273 & 0.254277 \\
\hline D81 & -0.270151 & 0.634695 & D205 & 0.154511 & -0.667953 \\
\hline D82 & 0.007987 & -0.148734 & D206 & -0.052644 & 0.016207 \\
\hline
\end{tabular}




\begin{tabular}{|c|c|c|c|c|c|}
\hline D83 & -0.386855 & 0.305541 & D207 & -0.267096 & 0.359409 \\
\hline D84 & -0.027249 & 0.070575 & D208 & 0.474524 & -0.785205 \\
\hline D85 & 0.288432 & -0.115569 & D209 & -0.129397 & 0.655329 \\
\hline D86 & -0.026944 & 0.420022 & D210 & 0.024233 & -0.148734 \\
\hline D87 & 0.17398 & -0.549783 & D211 & -0.369053 & 0.526679 \\
\hline D88 & 0.085655 & 0.122974 & D212 & -0.083173 & 0.121723 \\
\hline D89 & -0.182333 & 0.359409 & D213 & 0.346901 & -0.296621 \\
\hline D90 & -0.273259 & 0.634695 & D214 & 0.043303 & 0.444678 \\
\hline D91 & 0.233911 & -0.752716 & D215 & 0.233911 & -0.752716 \\
\hline D92 & 0.063935 & 0.015968 & D216 & 0.063935 & 0.015968 \\
\hline D93 & -0.376761 & 0.463675 & D217 & -0.376761 & 0.463675 \\
\hline D94 & 0.422932 & -0.719435 & D218 & 0.422932 & -0.719435 \\
\hline D95 & -0.135914 & 0.485793 & D219 & -0.135914 & 0.485793 \\
\hline D96 & 0.082961 & -0.295247 & D220 & 0.082961 & -0.295247 \\
\hline D97 & -0.30777 & 0.220657 & D221 & -0.30777 & 0.220657 \\
\hline D98 & -0.038983 & 0.081976 & D222 & -0.038983 & 0.081976 \\
\hline D99 & 0.185409 & -0.178454 & D223 & 0.185409 & -0.178454 \\
\hline D100 & 0.050333 & 0.422677 & D224 & 0.050333 & 0.422677 \\
\hline D101 & 0.171428 & -0.549783 & D225 & 0.171428 & -0.549783 \\
\hline D102 & -0.052644 & 0.016207 & D226 & -0.052644 & 0.016207 \\
\hline D103 & -0.256243 & 0.359409 & D227 & -0.256243 & 0.359409 \\
\hline D104 & 0.510496 & -0.785205 & D228 & 0.510496 & -0.785205 \\
\hline D105 & -0.094154 & 0.655329 & D229 & -0.094154 & 0.655329 \\
\hline D106 & -0.002407 & -0.148734 & D230 & -0.002407 & -0.148734 \\
\hline D107 & -0.326118 & 0.305541 & D231 & -0.326118 & 0.305541 \\
\hline D108 & -0.03887 & 0.070575 & D232 & -0.03887 & 0.070575 \\
\hline D109 & 0.1867 & -0.200452 & D233 & 0.1867 & -0.200452 \\
\hline D110 & -0.013968 & 0.438902 & D234 & -0.013968 & 0.438902 \\
\hline D111 & 0.152455 & -0.667953 & D235 & 0.152455 & -0.667953 \\
\hline D112 & -0.103693 & 0.016207 & D236 & -0.103693 & 0.016207 \\
\hline D113 & -0.281088 & 0.466178 & D237 & -0.281088 & 0.466178 \\
\hline D114 & 0.474341 & -0.700436 & D238 & 0.474341 & -0.700436 \\
\hline D115 & -0.270151 & 0.634695 & D239 & -0.270151 & 0.634695 \\
\hline D116 & 0.007987 & -0.148734 & D240 & 0.007987 & -0.148734 \\
\hline D117 & -0.386855 & 0.305541 & D241 & -0.386855 & 0.305541 \\
\hline D118 & -0.027249 & 0.070575 & D242 & -0.027249 & 0.070575 \\
\hline D119 & 0.288432 & -0.115569 & D243 & 0.288432 & -0.115569 \\
\hline D120 & -0.026944 & 0.420022 & D244 & -0.026944 & 0.420022 \\
\hline D121 & 0.17398 & -0.549783 & D245 & 0.17398 & -0.549783 \\
\hline D122 & 0.085655 & 0.122974 & D246 & 0.085655 & 0.122974 \\
\hline D123 & -0.182333 & 0.359409 & D247 & -0.182333 & 0.359409 \\
\hline D124 & -0.273259 & 0.634695 & D248 & -0.273259 & 0.634695 \\
\hline
\end{tabular}




\section{Copyrights}

Copyright for this article is retained by the author(s), with first publication rights granted to the journal.

This is an open-access article distributed under the terms and conditions of the Creative Commons Attribution license (http://creativecommons.org/licenses/by/3.0/). 